\title{
The Thermus thermophilus DEAD box helicase Hera contains a modified RNA recognition motif domain loosely connected to the helicase core
}

\author{
MARKUS G. RUDOLPH ${ }^{1,3}$ and DAGMAR KLOSTERMEIER ${ }^{2,3}$ \\ ${ }^{1}$ Department of Molecular Structural Biology, University of Göttingen, D-37077 Göttingen, Germany \\ ${ }^{2}$ Division of Biophysical Chemistry, Biozentrum, University of Basel, $\mathrm{CH}-4056$ Basel, Switzerland
}

\begin{abstract}
DEAD box family helicases consist of a helicase core that is formed by two flexibly linked RecA-like domains. The helicase activity can be regulated by $\mathrm{N}$ - or $\mathrm{C}$-terminal extensions flanking the core. Thermus thermophilus heat resistant RNA-dependent ATPase (Hera) is the first DEAD box helicase that forms a dimer using a unique dimerization domain. In addition to the dimerization domain, Hera contains a C-terminal RNA binding domain (RBD) that shares sequence homology only to uncharacterized proteins of the Deinococcus/Thermus group. The crystal structure of Hera_RBD reveals the fold of an altered RNA recognition motif (RRM) with limited structural homology to the RBD of the DEAD box helicase YxiN from Bacillus subtilis. Comparison with RRM/RNA complexes shows that a RNA binding mode different than that suggested for YxiN, but similar to U1A, can be inferred for Hera. The orientation of the RBD relative to the helicase core was defined in a second crystal structure of a Hera fragment including the C-terminal RecA domain, the dimerization domain, and the RBD. The structures allow construction of a model for the entire Hera helicase dimer. A likely binding surface for large RNA substrates that spans both RecA-like domains and the RBD is identified.
\end{abstract}

Keywords: RNA helicase; DEAD box protein; Hera; ATPase; RNA recognition motif

\section{INTRODUCTION}

RNA helicases of the DEAD box family couple ATP hydrolysis to rearrangements of RNA structures (Hilbert et al. 2009) and are involved in many facets of RNA metabolism. They share a core domain that is subdivided into two RecA-like domains (RecA_N and RecA_C) that contain all conserved helicase motifs (Gorbalenya and Koonin 1993) involved in nucleotide binding, RNA bind-

\footnotetext{
${ }^{3}$ The authors have no conflict of interest.

Abbreviations: RBD, RNA binding domain; RRM, RNA recognition motif; Hera, heat-resistant RNA-dependent ATPase; RMSD: rootmean-square deviation; RecA_N, N-terminal RecA-like domain; RecA_C, C-terminal RecA-like domain; Hera_RBD, Hera residues 424-510; Hera_208-510, Hera including the second RecA-like domain, the dimerization motif, and Hera_RBD.

Reprint requests to: Dagmar Klostermeier, Division of Biophysical Chemistry, Biozentrum, University of Basel, Klingelbergstrasse 70, CH4056 Basel, Switzerland; e-mail: dagmar.klostermeier@unibas.ch; fax: +41 61-267-21-89.

Article published online ahead of print. Article and publication date are at http://www.rnajournal.org/cgi/doi/10.1261/rna.1820009.
}

ing, and coupling of ATP hydrolysis to RNA unwinding. A flexible linker allows for very different juxtapositions of the RecA-like domains. RNA helicases undergo large conformational changes during their catalytic cycle (Theissen et al. 2008), and an array of open helicase conformations has been structurally characterized (Caruthers et al. 2000; Story et al. 2001; Cheng et al. 2005; Andersen et al. 2006). In a few instances, such as eIF4A-III in the exon junction complex (Andersen et al. 2006; Bono et al. 2006), the Drosophila Vasa RNA helicase (Sengoku et al. 2006), and Dbp5/Ddx19 (Collins et al. 2009; von Moeller et al. 2009), closed conformations were obtained in complex with short oligo-U nucleotides and a non-hydrolyzable ATP analog. DEAD box proteins in general display little sequence specificity for RNA. For a few representatives, structures formed by specific RNA sequences are specifically recognized by C-terminal RNA binding domains (RBD). Examples are the Escherichia coli RNA helicase DbpA and its homolog from Bacillus subtilis (YxiN), as well as Thermus thermophilus Hera. All proteins bind and unwind a helix adjacent to a stem-loop structure in 23S rRNA (Diges and 
Uhlenbeck 2001; Kossen et al. 2002; Linden et al. 2008). Instead of RNA specificity, additional domains flanking the helicase core may mediate nonspecific binding of RNA with high affinity. Examples are the splicing DEAD box proteins Mss116 from yeast and Cyt-19 from Neurospora crassa that bind structured RNA with high affinity via basic C-terminal domains and act as general RNA chaperones (Huang et al. 2005; Mohr et al. 2006; Tijerina et al. 2006; Grohman et al. 2007; Halls et al. 2007; Mohr et al. 2008).

The structure of the C-terminal RBD of the RNA helicase YxiN has been determined (Wang et al. 2006) and reveals an RNA recognition motif (RRM) comprising a central $\beta$-sheet, flanked by two $\alpha$-helices on one side. Mutagenesis studies suggest that RNA binding does not involve conserved aromatic residues on the exposed surface of the $\beta$-sheet, and indicate a noncanonical mode of RNA binding. Overall, the mode of RNA recognition by DEAD box protein RBDs is currently not understood, and a model for binding of large RNA substrates to RNA helicases and the structural basis of RNA sequence specificity is lacking. Furthermore, the positioning of RBDs in the context of the helicase remains elusive.

We determined two crystal structures of C-terminal fragments of Hera from $T$. thermophilus (termed Hera RBD and Hera $208-510$ ). Hera_RBD adopts the fold of a degenerated RRM. A linker sequence connects Hera_RBD to the dimerization domain and places it on top of the C-terminal RecA-like domain. Assembly of the entire Hera helicase dimer via structure-based alignment with RNA complexes of other RNA helicases results in a model that allows definition of a likely RNA binding surface extending from the helicase core to the RBD.

\section{RESULTS}

Full-length Hera from T. thermophilus strain HB27 is a DEAD box helicase (Fig. 1A) that binds fragments of $23 \mathrm{~S}$ rRNA and RNase P RNA via its C-terminal domain (Linden et al. 2008). The structure of the $\mathrm{N}$-terminal RecA-like domain in complex with AMP has previously been described (Rudolph et al. 2006). Recently, we determined the structures of Hera fragments encompassing the C-terminal RecA-like domain, and established Hera as a stable dimer both in crystals and in solution (Klostermeier and Rudolph 2009). These structures revealed the approximate location of an additional, almost spherical domain, the Hera RBD. The high-resolution structure of the RBD and its orientation relative to the helicase core, however, are currently unknown. We report here the structures of Hera_RBD and of Hera_208-510, which contains the C-terminal RecA-like domain, the dimerization domain, and Hera_RBD. The structures were refined to resolutions of $1.8 \AA$ and $2.8 \AA$, respectively. Data collection and refinement statistics are summarized in Table 1.
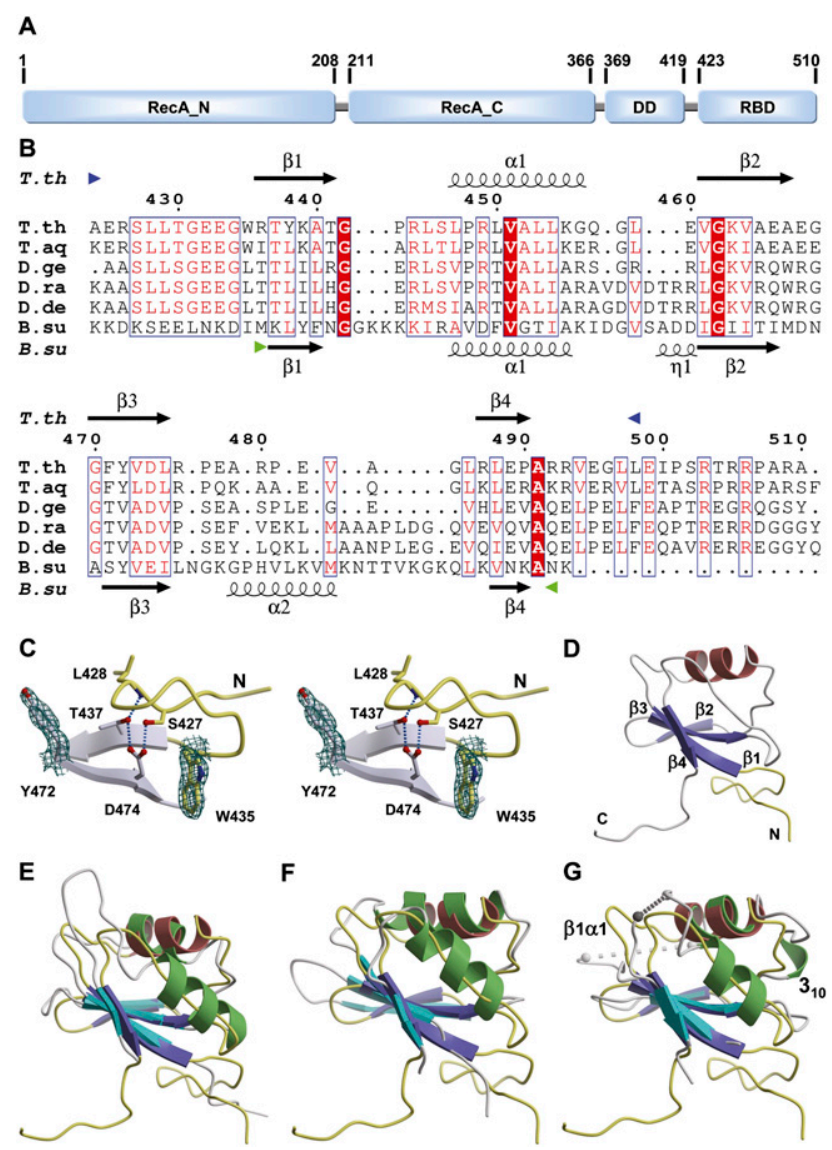

F
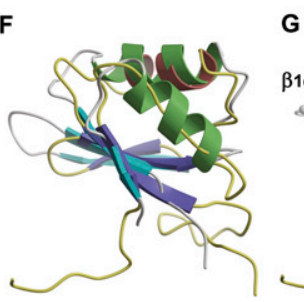

G

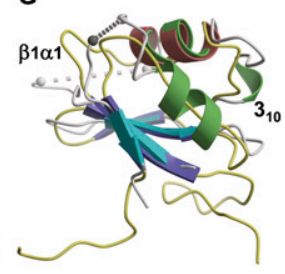

FIGURE 1. Hera_RBD structure sequence relationship and architecture. (A) Domain organization of Hera. The boxes match the domain sizes and the boundaries are indicated. Hera_RBD is located at the C terminus of Hera. The dimerization domain (DD) connects the RBD to the helicase core, which consists of two RecA-like domains termed RecA_N and RecA_C. (B) Sequence alignment of the T. thermophilus Hera RBD with putative helicases from T. aquaticus, D. geothermalis, $D$. radiodurans, $D$. deserti, and structure-based alignment with $B$. subtilis RNA helicase YxiN. The C-terminal sequences of the $T$. aquaticus and Deinococcus proteins have been truncated. The secondary structure elements of Hera_RBD are indicated on the top while those of the B. subtilis RBD are shown at the bottom for comparison. The sequence numbering corresponds to the full-length Hera. Blue and green arrows denote the sequence ranges that are visible in the Hera_RBD and YxiN structures. $(C)$ Stereo view of the small globular $\mathrm{N}$-terminal domain of Hera_RBD forming a hydrogen bond network with residues from $\beta 1$ and $\beta 3$. The experimental SIRAS electron density map is shown for Trp435 and Tyr472 at $2 \sigma$. The backbone trace of the small globular $\mathrm{N}$-terminal domain is shown in yellow. The side-chains are depicted as stick models colored according to atom type. $(D)$ Ribbon diagram of Hera_RBD with helix $\alpha 1$ in red and the $\beta$-strands in blue. The view is rotated $180^{\circ}$ about the $x$-axis compared to $C$. $(E-G)$ Comparison of Hera_RBD with other RRM structures. Hera_RBD is colored as in $C$. The $\alpha$-helices and $\beta$-strands of the compared structures are colored green and cyan, respectively. $(E)$ The best DALI hit is the human negative elongation factor $\mathrm{E}$ (Rao et al. 2006). (F) The closest structural homolog from a prokaryote is BT0572, a protein of unknown function from B. thetaiotaomicron. $(G)$ The structurally closest module from a RNA helicase is B. subtilis YxiN. The discontinuities in the YxiN structure are marked as spheres connected by dashed lines. Note the absence of the second $\alpha$-helix in Hera_RBD. 
Deinococcus radiodurans, and Deinococcus deserti (Fig. 1B) display a similarly shortened sequence as Hera. These bacteria belong to the Deinococcus/Thermus (D/T) group and are the only group in which a helicase with sequence homology with Hera has been identified (Linden et al. 2008). Most likely, these helicases have the same topological features in their C-terminal domains and share similar functions.

A search for structural homologs to Hera_RBD in the DALI database (http://www2.ebi.ac.uk/dali) returned 165 low significance hits with Z-scores between 3.5 and 6.1. Scores $<2.5$ are defined as structurally dissimilar and the self-score of a domain of equivalent size is $\sim 20$. Many of the identified proteins are involved in RNA binding and/or processing, and $2 / 3$ of all structures with DALI scores $>4.5$ are eukaryotic RRMs. The closest structural homolog is the human negative elongation factor E (Z-score 6.1; RMSD $2.7 \AA$ over 64 residues) that may be involved in transcriptional regulation of HIV-I (Fig. 1E; Rao et al. 2006). The closest prokaryotic homolog is BT0572 (Fig. 1F), a protein of unknown function from Bacteroides thetaiotaomicron (Z-score 5.2; RMSD $2.8 \AA$ over 62 residues; unpublished PDB-ID 2f06). The best match to a bacterial RNA helicase RRM is that of B. subtilis YxiN (Z-score of 4.5; RMSD $2.3 \AA$ over 51 residues) (Fig. 1G; Wang et al. 2006). The prime differences between Hera_RBD and the structures identified in the DALI search are the absence of helix $\alpha 2$ in Hera, but also the length of helix $\alpha 1$ and its angular disposition to the $\beta$-sheet. Of note, the ACT domain in T. thermophilus aspartokinase (Z-score 4.5; RMSD $3.4 \AA$ over 61 residues) shows comparable structural homology with Hera_RBD as the RRM domains discussed above. ACT domains are small molecule or protein binding entities in cytosolic proteins involved in metabolism, transport, and transcriptional regulation (Grant 2006). While ACT domains share the same topology as RRM, they are not involved in RNA binding. Taken together, Hera_RBD shares structural similarity with the RRM and ACT domains but differs significantly not only on the sequence, but also on the secondary structure level.

The RRM in Hera_RBD is flanked by sequences that are neither part of the dimerization domain nor flexible linkers between domains but adopt well-defined structures. The $\mathrm{N}$-terminal 12 residues 424-AERSLLTGEEGW-435 fold into a small globular structure containing two $\beta$-turns that attach to the central $\beta$-sheet of the RRM (Figs. 1C, 2A). Seven out of these 12 residues are identical in the $\mathrm{D} / \mathrm{T}$ group RNA helicases (noted in boldface). Hydrogen bonds are formed between the side-chains of Ser427 and Asp474, and between the amide nitrogen of Leu428 and Thr437. Asp474 and Thr437 of the RRM are also conserved, underscoring the importance of these interactions for $\mathrm{D} / \mathrm{T}$ group helicases. In addition, the conserved leucine residues 428 and 429 are engaged in hydrophobic interactions with the $\beta$-sheet and the sequence C-terminal to the RRM. In Hera_208-510, the small N-terminal motif mediates the contact of Hera_RBD with the C-terminal RecA domain (see below). Residues
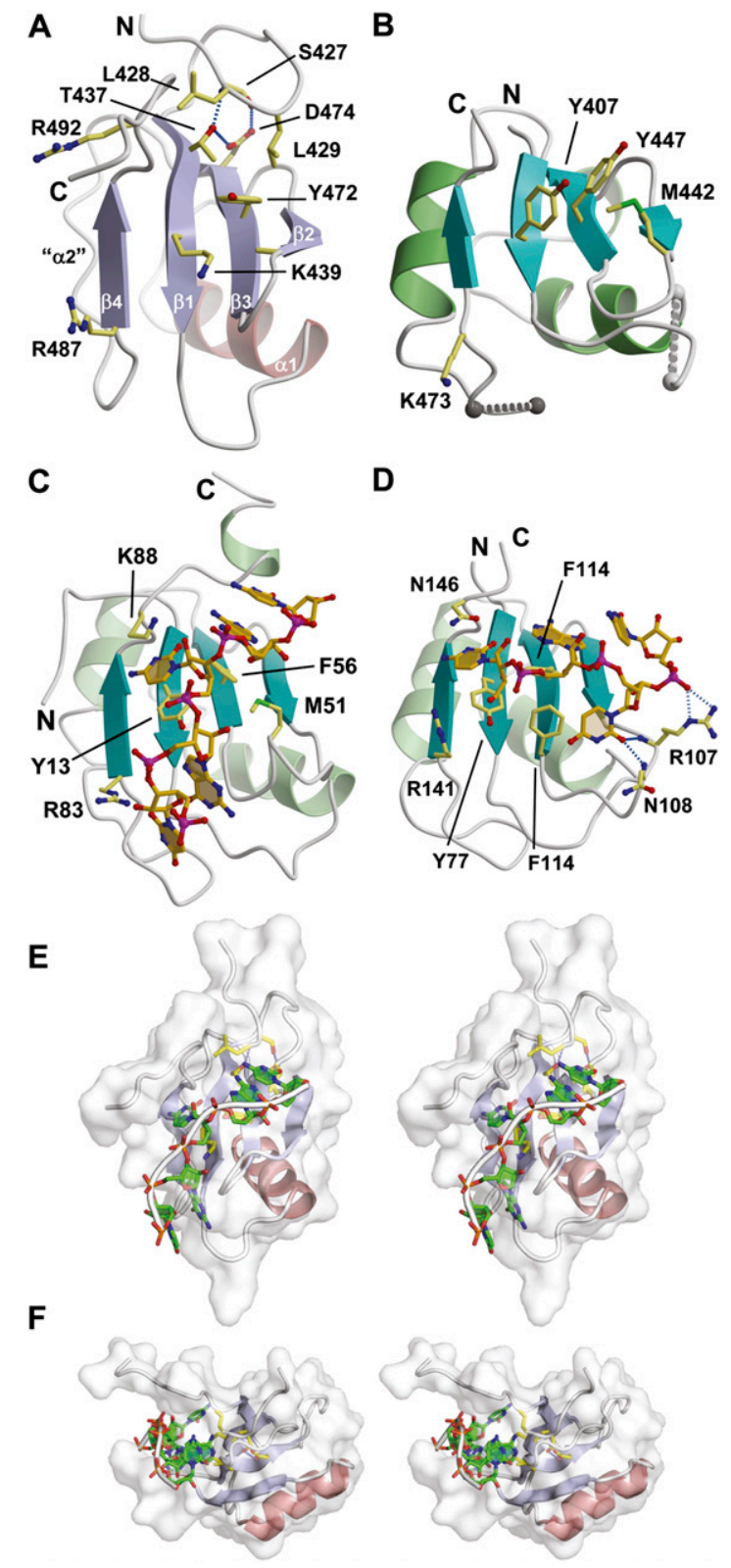

FIGURE 2. Comparison of Hera with other RRM domains and attempt to delineate the RNA binding mode. (A) Hera_RBD, $(B)$ YxiN. (C) U1A with RNA bound across the $\beta$-sheet. (D) SRp20 with RNA bound at the $\beta 2 / \beta 3$ loop. Residues on the $\beta$-sheet that bind RNA are drawn as stick models. Equivalent residues are drawn for Hera and YxiN for which the RNA binding modes are unknown. (E) Placement of the U1A-bound RNA from $C$ into a cleft of Hera_RBD. Hera_RBD is colored as in Figure 1D and the surface is rendered transparent. The U1A-RNA is shown as a stick model with carbon atoms colored green. $(F)$ View along the principal axis of the RNA.

492-RRVEGLLEIPSRTRRPARA-510 follow the Hera_RBD. Crystal contacts and interactions with the N-terminal motif noted above impose an extended conformation for the first seven residues (underscored) (Fig. 1D), while the rest are disordered. Several positively charged residues in this region 
may hint to unspecific RNA binding, but the sequence conservation among the $\mathrm{D} / \mathrm{T}$ group helicases is low.

\section{Comparison with YxiN and analysis of potential RNA binding surfaces in Hera_RBD}

Although there is no significant sequence homology between the two RBDs (Fig. 1B), a functional homology of YxiN and Hera in $23 S$ rRNA binding is likely. The $\beta 1 / \alpha 1$ and $\alpha 2 / \beta 4$ loop regions in YxiN contain various lysine residues and have been suggested to be involved in RNA binding (Wang et al. 2006). While the loops are disordered in YxiN and may only become ordered upon RNA binding, these loops have well-defined conformations in Hera_RBD (Fig. 2A,B). However, in Hera_RBD only a single positively charged residue, Arg444, is present in $\beta 1 / \alpha 1$ and no positive charges are present in $\alpha 2 / \beta 4$, rendering it unlikely for these loops to contribute to RNA binding to the same extent as suggested for YxiN. Thus, the RNA binding modes of Hera and YxiN may be very different.

RRMs in general utilize a variety of RNA binding modes (Maris et al. 2005; Dominguez and Allain 2006; Clery et al. 2008). The classical mode of RNA binding involves tyrosine or phenylalanine residues in $\beta 1$ and $\beta 3$ on the solventaccessible side of the $\beta$-sheet. The aromatic side-chains stack onto nucleobases and the RNA is bound diagonally across the $\beta$-sheet (Clery et al. 2008). This binding mode is exemplified in the complex of the splicesosomal U1A protein with RNA (Fig. 2C; Oubridge et al. 1994) or in complexes of human UP1 with several oligonucleotides (Myers and Shamoo 2004). A distinct RNA binding mode is present in the pre-mRNA splicing factor SRp20 (Hargous et al. 2006). SRp20, similar to the classical binding mode, binds RNA bases at the two aromatic residues in $\beta 1$ and $\beta 3$, but in addition contacts the RNA via a long $\beta 2 / \beta 3$ loop (Fig. 2D). As a consequence, the RNA is not bound diagonally across the $\beta$-sheet, but perpendicular to the strands at the lower half of the $\beta$-sheet. Furthermore, socalled quasi-RRMs have been described that presumably bind RNA exclusively via loops $\beta 1 / \alpha 1$ and $\beta 2 / \beta 3$, without contacts to residues of the $\beta$-sheet (Dominguez and Allain 2006). Quasi-RRMs contain a characteristic $\beta$-hairpin inserted in $\alpha 2 / \beta 4$ that may partake in RNA binding, but the structure of a quasi-RRM/RNA complex is currently unknown. Intermediate RNA binding modes to the ones described above exist, pointing to a whole spectrum of RNA-RRM positioning.

In order to define the probable RNA binding site on Hera_RBD, we compared the poses of RNA molecules in known RRM/RNA complexes with the surface properties of Hera_RBD. Superposition of the SRp20/RNA complex with Hera_RBD shows severe clashes of the RNA with the (shorter) $\beta 2 / \beta 3$ loop in Hera. The conformations of the $\beta 2 / \beta 3$ loops differ by up to $8 \AA$, rendering such an RNA binding mode unlikely for Hera. In contrast, it appears that the RNA bound across the $\beta$-sheet of U1A can be positioned onto Hera_RBD with only minor clashes that may be rectified by slight conformational adjustments (Fig. 2E,F). The U1A-like RNA binding mode would place nucleobases in close proximity to Lys439 in $\beta 1$ and Tyr472 in $\beta 3$ in Hera_RBD. These residues are analogous to the two aromatic residues on the $\beta$-sheet surface of canonical RRMs. Interactions between the aliphatic sidechain of Lys439 and the aromatic systems of nucleobases are favorable, and in principle these residues could allow for a classical RNA interaction mode similar to U1A.

The Hera_RBD side-chains Lys439 and Tyr472 have their equivalents Tyr407 and Tyr447 in YxiN. Mutagenesis of these tyrosines to alanine did not alter affinity for a 32mer RNA, apparently excluding their involvement in RNA binding (Wang et al. 2006). Together with the differences in the $\beta 1 / \alpha 1$ and $\alpha 2 / \beta 4$ loop regions noted above, different RNA binding modes of Hera and YxiN may be assumed. This brings up the question as to why YxiN does not use the canonical $\beta$-sheet residues for RNA binding, whereas Hera may bind RNA similar to U1A. A close inspection of the RBD structures shows that Tyr447 in YxiN adopts a gauche plus rotamer, which is a non-binding competent side-chain conformation that prevents stacking onto the nucleobases (Fig. 2B). The gauche plus rotamer of Tyr447 is dictated by the nearby Met 442 in the $\beta 2 / \beta 3$ loop and is stabilized by Thr440. In contrast, Tyr472 in Hera adopts the gauche minus conformation (Fig. 2A), which is the RNA binding competent conformation in all RNA complexes. Two aspects force Tyr472 into the gauche minus conformation: First, a Tyr472 gauche plus rotamer is impossible in Hera due to sterical hindrance by Leu429 in the small N-terminal domain in Hera_RBD. Second, the small side-chain of Ala467 in Hera does not produce a stereochemical clash with Tyr472, as the equivalent Met442 does for Tyr447 in YxiN. Consequently, Tyr472 in Hera is stabilized in the conformation compatible with a canonical U1A-like RNA binding mode, whereas this binding mode is not possible for YxiN. In summary, a binding mode similar to U1A can be postulated for Hera_RBD, and different RNA binding modes for Hera and YxiN can be rationalized from the structures.

\section{Placement of the RBD in the context of Hera}

The RBDs provide DEAD box proteins with high affinity RNA binding of either high specificity for dedicated helicases (Kossen et al. 2002; Linden et al. 2008), or low specificity for helicases that are considered to function as general RNA chaperones (Mohr et al. 2008; Russell 2008; Del Campo et al. 2009). A role of RBDs in positioning neighboring RNA regions for unwinding by the helicase core has been suggested (Diges and Uhlenbeck 2005; Tijerina et al. 2006). While we have previously reported the global localization of the Hera_RBD with respect to the 
C-terminal RecA-like domain (Klostermeier and Rudolph 2009), the tracing of the RBD sequence was impossible. We therefore determined the structure of Hera_208-510 that comprises the C-terminal RecA-like domain, the dimerization motif, and the RBD (Fig. 3A). Molecular replacement of Hera_208-510 with Hera_208-419 and Hera_RBD resulted in a model where a monomer is present in the asymmetric unit, and a dimer is produced by crystal symmetry. The dimerization domain in Hera_208-510 is followed by a low complexity linker sequence (GGAPA) that connects the dimerization motif to the RBD. In the structure, this linker sequence traverses the upper part of the RecA_C domain and, as a result, loosely connects the RBD to RecA_C (Fig. 3A, cyan). Only a few polar and hydrophobic, but no hydrogen bond interactions attach the RBD to RecA_C: An electrostatic interaction between Glu227 in RecA_C and Arg426 in the RBD is present, and a total of only 17 van der Waals contacts connect the RBD to RecA_C. Residues participating in hydrophobic interactions are Pro221, Arg223, Gly224, and Arg345 of RecA_C, and residues Arg426, Ser427, Leu428, Thr430, Gly431, Lys463, and Ala465 of Hera_RBD. The buried surface area between the domains covers a mere $435 \AA^{2}$. Notably, most hydrophobic contacts are mediated by residues forming the small globular motif at the $\mathrm{N}$ terminus of Hera_RBD (Fig. 3A,B, yellow). The overall positioning of the RBD is with the central $\beta$-sheet facing away from RecA_C, leaving a small cleft between the domains. Due to
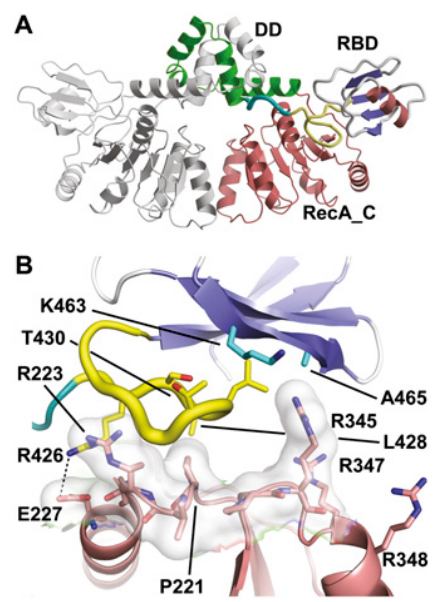

FIGURE 3. Location of the C-terminal domain in Hera. (A) Ribbon diagram of the refined Hera_208-510 structure. The second protomer of the dimer (gray) is generated by crystal symmetry, placing the Hera_RBDs at a distance of $75 \AA$. The linker connecting the dimerization domain (DD) to the RBD is shown in cyan and the $\mathrm{N}$-terminal motif of the RBD mediating contact with RecA_C is shown as a yellow tube. $(B)$ Magnification of the contact area between the RBD and RecA_C. Residues of RecA_C contributing to van der Waals interaction with the RBD are shown in pink with their transparent surface. Residues of the RBD contacting RecA_C are shown as yellow or cyan stick models. The polar interaction between Arg426 of the RBD and E227 of RecA_C is indicated by a dashed line. the likely flexibility of the linker region between the dimerization domain and the RBD, the RBD may adopt several conformations in solution that may be linked to RNA unwinding by the helicase core.

\section{DISCUSSION}

In the present work, we have determined the crystal structure of the isolated Hera RNA binding domain. In a second structure, the orientation of Hera_RBD within the framework of the entire helicase was defined. Hera_RBD forms a strongly altered RRM where an entire $\alpha$-helix is replaced by a loop region. Sequence comparison reveals that this structural motif should be conserved in the $\mathrm{D} / \mathrm{T}$ group of bacteria. This region is located on the "backside" of the $\beta$-sheet and therefore probably not involved in RNA binding, but it may have a structural role.

The N-terminal sequence of Hera_RBD that precedes the RRM is strongly conserved among the D/T DEAD box helicases and will form the same double- $\beta$-hairpin structure in all cases, possibly contributing to stabilization of the RRM. The negatively charged motif packs against one end of the $\beta$-sheet and could block RNA binding in this region, limiting the conformational space accessible to large RNA substrates. Residues from this region interact with residues of the RRM that are conserved in the $\mathrm{D} / \mathrm{T}$ group but not in DEAD-box helicases from mesophilic bacteria. N-terminal extensions are also present in YxiN and DbpA, but their structures are unknown. However, these extensions have negatively charged residues in common, which may similarly restrict RNA access to this side of the RRM. An $\mathrm{N}$-terminal extension is also present in the RRM of the U11/U12-65K spliceosomal protein, which binds to a hairpin of the U12 snRNA (Netter et al. 2009). However, in contrast to Hera, this motif of ca. 30 residues folds into a $\alpha$-helical structure. Deletion of the motif destabilizes the interaction of the U11/U12-65K RRM with RNA, and a structure-stabilizing effect was attributed to this motif (Netter et al. 2009).

Despite the lack of structural data on RNA binding to Hera, a canonical RNA binding mode resembling that of U1A is likely. Sequence comparisons of RRM domains show that deviations from the canonical RNA binding motifs RNP-1 and RNP-2 are common. Hera underscores this observation and adds the possibility that the aromatic side-chain in RNP-2 used for base-stacking can be replaced by aliphatic residues, namely lysine in the Thermus and isoleucine in the Deinococcus species. Even the aromatic side-chain in RNP-1 is not conserved but can be replaced by valine in Deinococci (Fig. 1B). In conclusion, the use of noncanonical RNA binding motifs emphasizes the plasticity of the RRMs not only in eukaryotes but also in prokaryotes.

Comparison of the different poses of RNA in RRM/RNA complexes with Hera_RBD has shown that Hera_RBD could bind RNA in a manner similar to U1A. U1A binds 
to a hairpin structure in U1 snRNA and specifically recognizes bases in the loop region. A similar binding mode of Hera to the loop region of hairpin 92 of the 23S rRNA can be envisaged, which would place the sequence $5^{\prime}$-UGUUC-3' across the $\beta$-sheet. In this configuration, the stem of the hairpin would project away from the RBD, leaving room for the remainder of the RNA substrate to contact the helicase core. Hera then unwinds a doublestranded region $5^{\prime}$ to the hairpin structure, and the RBD could help to align the RNA substrate for unwinding by the helicase core. In contrast to YxiN which displays a high specificity for hairpin 92 in 23S rRNA, Hera is promiscuous and also binds to RNase P RNA (Linden et al. 2008). The possible positioning function combined with substrate promiscuity would thus be analogous to the role of the basic domains in Mss116 and Cyt-19 (Mohr et al. 2008), that mediate nonspecific binding to a multitude of RNA structures (Huang et al. 2005; Mohr et al. 2006; Grohman et al. 2007; Halls et al. 2007). These basic CTDs preferentially interact with structured RNAs, and the helicase unwinds adjacent duplexes that are not tightly interacting with the structured part of the RNA (Tijerina et al. 2006).

While the dynamic interplay between the helicase core and the RBD is currently unknown, the crystal structures described here shed light on the juxtaposition of the Hera domains in the complete structure. The structure of the Vasa helicase core in the closed conformation (Sengoku et al. 2006) was used as a template to assemble a model for the dimeric Hera helicase from the individual domains (Fig. 4A; Rudolph et al. 2006; this work). The RNA cocrystallized with Vasa is short and only contacts the helicase core. The overall RNA binding mode of the helicase core is conserved and, if Hera_RBD binds RNA similarly to U1A, two pieces of RNA can be placed on the dimer: one bound across the core domain interface, and the second bound to the RBD (Fig. 4A). The directionality of these RNA molecules is the same, and the $5^{\prime}$-end of the core-bound RNA points toward the RBD. Electrostatic surface potential calculations of the Hera model identified an elongated, positively polarized region generated by arginine residues 225,246 , and 314 that starts $5^{\prime}$ of the poly-U binding site of Vasa and stretches via RecA_C toward the RBD. A positively charged ridge is present close to the RBD, formed by arginines 223, 345, 347, and 348 (Fig. 4B). These residues were suggested as possible RNA interacting sidechains (Klostermeier and Rudolph 2009), and passing of the RNA over this ridge on RecA_C to the RNA interacting with the RBD could be envisaged. There is also positive potential at the $3^{\prime}$ end of the bound RNA, possibly extending the RNA binding surface on RecA_N. The entire path could mark an elongated, unspecific ssRNA binding site. An extended binding site involving the helicase core and a C-terminal domain is observed in a recent crystal structure of Mss116 in complex with U10 ssRNA (Del Campo and Lambowitz 2009). The RNA bound to
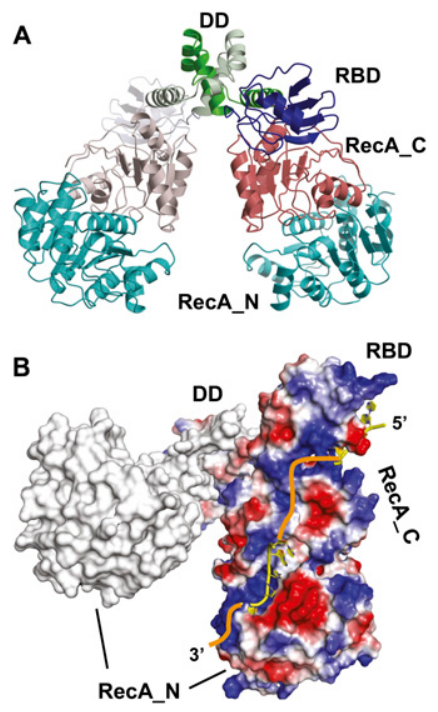

FIGURE 4. Possible binding site on Hera for large RNA molecules. (A) Construction of a model for the Hera dimer by superposition of the Hera_N (PDB-ID 1gxs) and Hera_208-510 (this work) structures with the Vasa-RNA complex. The domains are individually colored and labeled. The domains of the second monomer are colored in a lighter hue. The view is rotated by $45^{\circ}$ about the $y$-axis compared to Figure $3 \mathrm{~A}$ and the RBD faces the viewer. $(B)$ The surface electrostatic potential of the Hera dimer shows a positively polarized trace between the known RNA binding site on the helicase core and the RBD that could mark the path of a large RNA substrate (marked in orange). The RNAs were taken from the superposition with Vasa and U1A and are shown as stick models.

Mss116 has the same orientation as proposed for Hera and the $5^{\prime}$-end contacts a four-helix bundle C-terminal to the helicase core. In Hera, at least 25 nucleotides (nt) of ssRNA could be spanned across a monomer if the RNA followed the positive path. The dimer may therefore interact with $50 \mathrm{nt}$ or more, possibly in the context of larger RNA substrates.

\section{MATERIALS AND METHODS}

\section{Protein production, purification, and crystallization}

Hera_208-510 was cloned, produced, purified, crystallized, and MAD-phased as described (Rudolph et al. 2009). As the C-terminal 90 residues could not be traced, the region coding for Hera_RBD was cloned separately into pETM30. The protein was overproduced in E. coli Rosetta(DE3) in autoinducing medium (Studier 2005), and cells were disrupted in $50 \mathrm{mM}$ Tris/ $\mathrm{HCl}$ at $\mathrm{pH} 7.5$ and $500 \mathrm{mM} \mathrm{NaCl}$ using a microfluidizer. The supernatant was purified via affinity chromatography on GSHsepharose in the same buffer, and eluted with buffer containing 20 $\mathrm{mM}$ glutathione. After hydrolysis with TEV protease $\left(4^{\circ} \mathrm{C}\right.$, overnight), the remaining fusion protein and the $\mathrm{His}_{6}$-GST tag were removed using $\mathrm{Ni}^{2+}$-NTA agarose $(50 \mathrm{mM}$ Tris/ $\mathrm{HCl}$ at $\mathrm{pH} 7.5$, $500 \mathrm{mM} \mathrm{NaCl}, 20 \mathrm{mM}$ imidazole), followed by S75 size-exclusion chromatography (50 mM Tris/ $\mathrm{HCl}$ at $\mathrm{pH} 7.5,500 \mathrm{mM} \mathrm{NaCl})$. Hera_RBD was concentrated to $1.4 \mathrm{mM}$ in $50 \mathrm{mM}$ Tris/ $\mathrm{HCl}$ at $\mathrm{pH}$ 
7.5, $500 \mathrm{mM} \mathrm{NaCl}$, and diluted threefold with water prior to crystallization. Crystals were obtained after two weeks at $22^{\circ} \mathrm{C}$ via vapor diffusion in sitting drops by mixing equal volumes $(0.3 \mu \mathrm{L})$ of protein with reservoir solution consisting of $0.1 \mathrm{M} \mathrm{Na}$ cacodylate at $\mathrm{pH}$ 6.5, 16\%-18\% PEG8000, $0.3 \mathrm{M}-0.4 \mathrm{M}$ $\mathrm{Zn}(\mathrm{OAc})_{2}$. A crystal was derivatized using a 1-min soak with $0.25 \mathrm{M} \mathrm{KI}$ in reservoir solution. Prior to data collection, crystals were vitrified in paraffin oil using the hyper-quenching technique (Warkentin and Thorne 2007).

\section{Data collection, structure determination, and refinement}

Hera_RBD crystals are of space group $\mathrm{P} 4{ }_{1} 2_{1} 2$ and contain one molecule per asymmetric unit. Data were collected at $100 \mathrm{~K}$ and reduced with XDS (Kabsch 1993) and SADABS (Bruker). Native data were collected on a Pilatus detector on SLS beamline X06SA to a resolution of $1.8 \AA$. KI derivative data were collected to a resolution of $2.14 \AA$ on a MAR-CCD225 detector on beamline X06DA using a wavelength of $1.8 \AA$, where the calculated $\mathrm{f}^{\prime}=$ $-1.1 \mathrm{e}^{-}$and $\mathrm{f}^{\prime \prime}=8.8 \mathrm{e}^{-}$. For SIRAS phasing, the anomalous and SIR differences were extracted by XPREP (Bruker) using data to a resolution of $2.5 \AA$. SHELXD was used to determine a three-atom substructure. Enantiomorph selection and density modification including phase extension to $1.8 \AA$ resolution were performed with SHELXE, which also provided a first auto-traced polyalanine model of 73 out of 87 residues. The three iodides bind to the side-chain of R476 $(8.7 \sigma)$, between the side-chains of R449 and P443 $(8.1 \sigma)$, and to the main-chain amide groups of L497 and L498 $(5.5 \sigma)$. It should be noted that exhaustive molecular replacement searches using the MAD electron density (Klostermeier and Rudolph 2009), all available RRM-like models (including the YxiN homolog), or models calculated de novo from the Hera_RBD sequence using ROSETTA (Das and Baker 2008) were unsuccessful. Also unsuccessful were attempts to MAD-phase the crystals using data from a $\mathrm{KBr}$ soak or chemically modifying the two tyrosine side-chains in Hera_RBD using elementary iodine. The Hera_208-510 structure was determined via molecular replacement using the Hera_208-419 (Klostermeier and Rudolph 2009) and Hera_RBD structures as starting models with PHASER (McCoy et al. 2007). Models were built in COOT (Emsley and Cowtan 2004) and refined with PHENIX (Zwart et al. 2008). Five percent of reflections were reserved for $\mathrm{R}_{\text {free }}$ cross-validation in all structures (Brünger 1992). The C-terminal 12 residues (sequence EIPSRTRRPARA) were omitted from the final models due to lack of electron density. Statistics are summarized in Table 1. Figures were created with Bobscript (Esnouf 1997), Raster3D (Merritt and Murphy 1994), PyMol (http://www.pymol.org), or ESPRIPT (Gouet et al. 1999).

\section{DATA DEPOSITION}

The coordinates and structure factors have been deposited in the Protein Data Bank (accession codes 3I31 and 3I32).

\section{SUPPLEMENTAL MATERIAL}

Supplemental material can be found at http://www.rnajournal.org.

\section{ACKNOWLEDGMENTS}

We thank Ines Hertel for excellent technical assistance, and the colleagues at the Swiss Light Source for beamtime and guidance. This work was supported by grants from the DFG (to M.G.R.), the VolkswagenStiftung (to D.K.), and the Swiss National Science Foundation (to D.K.).

Received July 13, 2009; accepted July 31, 2009.

\section{REFERENCES}

Andersen CB, Ballut L, Johansen JS, Chamieh H, Nielsen KH, Oliveira CL, Pedersen JS, Seraphin B, Le Hir H, Andersen GR. 2006. Structure of the exon junction core complex with a trapped DEAD-box ATPase bound to RNA. Science 313: 1968-1972.

Bono F, Ebert J, Lorentzen E, Conti E. 2006. The crystal structure of the exon junction complex reveals how it maintains a stable grip on mRNA. Cell 126: 713-725.

Brünger AT. 1992. Free R value: A novel statistical quantity for assessing the accuracy of crystal structures. Nature 355: 472-475.

Caruthers JM, Johnson ER, McKay DB. 2000. Crystal structure of yeast initiation factor 4A, a DEAD-box RNA helicase. Proc Natl Acad Sci 97: 13080-13085.

Cheng Z, Coller J, Parker R, Song H. 2005. Crystal structure and functional analysis of DEAD-box protein Dhhlp. RNA 11: 12581270.

Clery A, Blatter M, Allain FH. 2008. RNA recognition motifs: Boring? Not quite. Curr Opin Struct Biol 18: 290-298.

Collins R, Karlberg T, Lehtio L, Schutz P, van den Berg S, Dahlgren LG, Hammarstrom M, Weigelt J, Schuler H. 2009. The DEXD/H-box RNA helicase DDX19 is regulated by an $\alpha$-helical switch. J Biol Chem 284: 10296-10300.

Das R, Baker D. 2008. Macromolecular modeling with rosetta. Annu Rev Biochem 77: 363-382.

Del Campo M, Lambowitz AM. 2009. Structure of the yeast DEADbox protein Mss116p reveals two wedges that crimp RNA. Mol Cell (in press).

Del Campo M, Mohr S, Jiang Y, Jia H, Jankowsky E, Lambowitz AM. 2009. Unwinding by local strand separation is critical for the function of DEAD-box proteins as RNA chaperones. J Mol Biol 389: 674-693.

Diges CM, Uhlenbeck OC. 2001. Escherichia coli DbpA is an RNA helicase that requires hairpin 92 of $23 \mathrm{~S}$ rRNA. EMBO J 20: 55035512.

Diges CM, Uhlenbeck OC. 2005. Escherichia coli DbpA is a $3^{\prime} \rightarrow 5^{\prime}$ RNA helicase. Biochemistry 44: 7903-7911.

Dominguez C, Allain FH. 2006. NMR structure of the three quasi RNA recognition motifs (qRRMs) of human hnRNP $F$ and interaction studies with Bcl-x G-tract RNA: A novel mode of RNA recognition. Nucleic Acids Res 34: 3634-3645.

Emsley P, Cowtan K. 2004. Coot: Model-building tools for molecular graphics. Acta Cryst D60: 2126-2132.

Esnouf RM. 1997. An extensively modified version of MOLSCRIPT that includes greatly enhanced coloring capabilities. J Mol Graph 15: $132-134$.

Gorbalenya AE, Koonin EV. 1993. Helicases: Amino acid sequence comparisons and structure-function relationships. Curr Opin Struct Biol 3: 419-429.

Gouet P, Courcelle E, Stuart DI, Metoz F. 1999. ESPript: Analysis of multiple sequence alignments in PostScript. Bioinformatics 15: 305-308.

Grant GA. 2006. The ACT domain: A small molecule binding domain and its role as a common regulatory element. J Biol Chem 281: 33825-33829.

Grohman JK, Del Campo M, Bhaskaran H, Tijerina P, Lambowitz AM, Russell R. 2007. Probing the mechanisms of 
DEAD-box proteins as general RNA chaperones: The C-terminal domain of CYT-19 mediates general recognition of RNA. Biochemistry 46: 3013-3022.

Halls C, Mohr S, Del Campo M, Yang Q, Jankowsky E, Lambowitz AM. 2007. Involvement of DEAD-box proteins in group I and group II intron splicing. Biochemical characterization of Mss116p, ATP hydrolysis-dependent and -independent mechanisms, and general RNA chaperone activity. J Mol Biol 365: 835-855.

Hargous Y, Hautbergue GM, Tintaru AM, Skrisovska L, Golovanov AP, Stevenin J, Lian LY, Wilson SA, Allain FH. 2006. Molecular basis of RNA recognition and TAP binding by the SR proteins SRp20 and 9G8. EMBO J 25: 5126-5137.

Hilbert M, Karow AR, Klostermeier D. 2009. The mechanism of ATPdependent RNA unwinding by DEAD-box proteins. Biol Chem (in press).

Huang HR, Rowe CE, Mohr S, Jiang Y, Lambowitz AM, Perlman PS. 2005. The splicing of yeast mitochondrial group I and group II introns requires a DEAD-box protein with RNA chaperone function. Proc Natl Acad Sci 102: 163-168.

Kabsch W. 1993. Automatic processing of rotation diffraction data from crystals of initially unknown symmetry and cell constants. J Appl Crystallogr 26: 795-800.

Klostermeier D, Rudolph MG. 2009. A novel dimerization motif in the C-terminal domain of the Thermus thermophilus DEAD box helicase Hera confers substantial flexibility. Nucleic Acids Res 37: 421-430.

Kossen K, Karginov FV, Uhlenbeck OC. 2002. The carboxy-terminal domain of the DExDH protein YxiN is sufficient to confer specificity for 23S rRNA. J Mol Biol 324: 625-636.

Linden MH, Hartmann RK, Klostermeier D. 2008. The putative RNase P motif in the DEAD box helicase Hera is dispensable for efficient interaction with RNA and helicase activity. Nucleic Acids Res 36: $5800-5811$.

Maris C, Dominguez C, Allain FH. 2005. The RNA recognition motif, a plastic RNA-binding platform to regulate post-transcriptional gene expression. FEBS J 272: 2118-2131.

McCoy AJ, Grosse-Kunstleve RW, Adams PD, Winn MD, Storoni LC, Read RJ. 2007. Phaser crystallographic software. J Appl Crystallogr 40: 658-674.

Merritt EA, Murphy MEP. 1994. Raster3D Version 2.0: A program for photorealistic molecular graphics. Acta Crystallogr D Biol Crystal$\log r$ 50: 869-873.

Mohr S, Matsuura M, Perlman PS, Lambowitz AM. 2006. A DEADbox protein alone promotes group II intron splicing and reverse splicing by acting as an RNA chaperone. Proc Natl Acad Sci 103: 3569-3574.

Mohr G, Del Campo M, Mohr S, Yang Q, Jia H, Jankowsky E, Lambowitz AM. 2008. Function of the C-terminal domain of the DEAD-box protein Mss116p analyzed in vivo and in vitro. $J \mathrm{Mol}$ Biol 375: 1344-1364.

Myers JC, Shamoo Y. 2004. Human UP1 as a model for understanding purine recognition in the family of proteins containing the RNA recognition motif (RRM). J Mol Biol 342: 743-756.
Netter C, Weber G, Benecke H, Wahl MC. 2009. Functional stabilization of an RNA recognition motif by a noncanonical N-terminal expansion. RNA 15: 1305-1313.

Oubridge C, Ito N, Evans PR, Teo CH, Nagai K. 1994. Crystal structure at $1.92 \AA$ resolution of the RNA-binding domain of the U1A spliceosomal protein complexed with an RNA hairpin. Nature 372: 432-438.

Rao JN, Neumann L, Wenzel S, Schweimer K, Rosch P, Wohrl BM. 2006. Structural studies on the RNA-recognition motif of NELF E, a cellular negative transcription elongation factor involved in the regulation of HIV transcription. Biochem J 400: 449-456.

Rudolph MG, Heissmann R, Wittmann JG, Klostermeier D. 2006. Crystal structure and nucleotide binding of the Thermus thermophilus RNA helicase Hera N-terminal domain. J Mol Biol 361: 731743.

Rudolph MG, Wittmann JG, Klostermeier D. 2009. Crystallization and preliminary characterization of the Thermus thermophilus RNA helicase Hera C-terminal domain. Acta Crystallogr Sect F Struct Biol Cryst Commun 65: 248-252.

Russell R. 2008. RNA misfolding and the action of chaperones. Front Biosci 13: 1-20.

Sengoku T, Nureki O, Nakamura A, Kobayashi S, Yokoyama S. 2006. Structural basis for RNA unwinding by the DEAD-box protein Drosophila Vasa. Cell 125: 287-300.

Story RM, Li H, Abelson JN. 2001. Crystal structure of a DEAD box protein from the hyperthermophile Methanococcus jannaschii. Proc Natl Acad Sci 98: 1465-1470.

Studier FW. 2005. Protein production by auto-induction in high density shaking cultures. Protein Expr Purif 41: 207-234.

Theissen B, Karow AR, Kohler J, Gubaev A, Klostermeier D. 2008. Cooperative binding of ATP and RNA induces a closed conformation in a DEAD box RNA helicase. Proc Natl Acad Sci 105: 548553.

Tijerina P, Bhaskaran H, Russell R. 2006. Nonspecific binding to structured RNA and preferential unwinding of an exposed helix by the CYT-19 protein, a DEAD-box RNA chaperone. Proc Natl Acad Sci 103: 16698-16703.

von Moeller H, Basquin C, Conti E. 2009. The mRNA export protein DBP5 binds RNA and the cytoplasmic nucleoporin NUP214 in a mutually exclusive manner. Nat Struct Mol Biol 16: 247-254.

Wang S, Hu Y, Overgaard MT, Karginov FV, Uhlenbeck OC, McKay DB. 2006. The domain of the Bacillus subtilis DEAD-box helicase YxiN that is responsible for specific binding of $23 \mathrm{~S}$ rRNA has an RNA recognition motif fold. RNA 12: 959-967.

Warkentin M, Thorne RE. 2007. A general method for hyperquenching protein crystals. J Struct Funct Genomics 8: 141-144.

Zwart PH, Afonine PV, Grosse-Kunstleve RW, Hung LW, Ioerger TR, McCoy AJ, McKee E, Moriarty NW, Read RJ, Sacchettini JC, et al. 2008. Automated structure solution with the PHENIX suite. Methods Mol Biol 426: 419-435. 

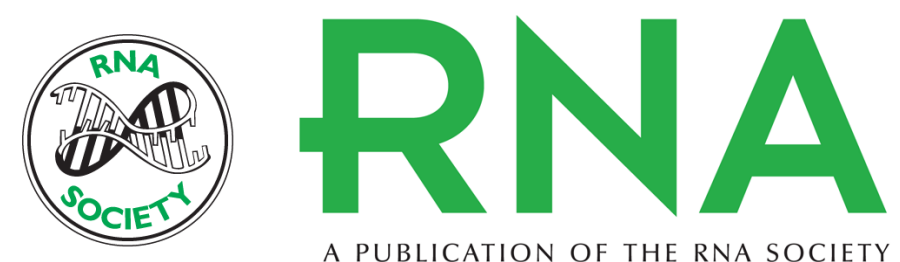

A PUBLICATION OF THE RNA SOCIETY

\section{The Thermus thermophilus DEAD box helicase Hera contains a modified RNA recognition motif domain loosely connected to the helicase core}

Markus G. Rudolph and Dagmar Klostermeier

RNA 2009 15: 1993-2001 originally published online August 26, 2009

Access the most recent version at doi:10.1261/rna.1820009

Supplemental Material

References

License

Email Alerting Service
http://rnajournal.cshlp.org/content/suppl/2009/08/24/rna.1820009.DC1

This article cites 46 articles, 14 of which can be accessed free at: http://rnajournal.cshlp.org/content/15/11/1993.full.html\#ref-list-1

Receive free email alerts when new articles cite this article - sign up in the box at the top right corner of the article or click here. 